\title{
Stationary reflection in extender models
}

\author{
by
}

Ernest Schimmerling (Pittsburgh, PA)

\begin{abstract}
Working in $L[E]$, we examine which large cardinal properties of $\kappa$ imply that all stationary subsets of $\operatorname{cof}(<\kappa) \cap \kappa^{+}$reflect.
\end{abstract}

1. Introduction. Suppose that $\kappa$ is an infinite cardinal and $\tau<\kappa^{+}$is a limit ordinal. Let $A$ be stationary in $\kappa^{+}$. Then $A$ reflects to $\tau$ if $A \cap \tau$ is stationary in $\tau$. The case in which $\tau$ has countable cofinality is degenerate, so we ignore it for the most part. Also, only $\tau>\kappa$ are ultimately relevant. Define

$$
\begin{aligned}
\operatorname{cof}(\kappa) & =\{\alpha \in \mathrm{OR} \mid \operatorname{cf}(\alpha)=\kappa\}, \\
\operatorname{cof}(<\kappa) & =\{\alpha \in \mathrm{OR} \mid \operatorname{cf}(\alpha)<\kappa\} .
\end{aligned}
$$

Notice that if $\kappa$ is a regular cardinal, then $\kappa^{+} \cap \operatorname{cof}(\kappa)$ is stationary in $\kappa^{+}$but does not reflect. Stationary Reflection at $\kappa^{+}$asserts that for all $A \subseteq \kappa^{+} \cap \operatorname{cof}(<\kappa)$, if $A$ is stationary, then $A$ reflects. It is well known that if $\square_{\kappa}$ holds, then every stationary subset of $\kappa^{+}$has a non-reflecting stationary subset. This consequence of $\square_{\kappa}$ is known as Dense Non-Reflection at $\kappa^{+}$.

In this report, $L[E]$ always stands for a Jensen style extender model. It is known that in an extender model,

$$
\kappa \text { is a subcompact cardinal iff } \square_{\kappa} \text { fails. }
$$

We will explain credit for this result when we discuss it in more detail later. But we should recall one definition without delay.

Definition $1.1([3]) . \kappa$ is a subcompact cardinal iff for all $A \subseteq \kappa^{+}$, there exist $\mu<\kappa, a \subseteq \mu^{+}$and an elementary embedding

$$
\pi:\left(H_{\mu^{+}}, a\right) \rightarrow\left(H_{\kappa^{+}}, A\right)
$$

with $\operatorname{crit}(\pi)=\mu$.

2000 Mathematics Subject Classification: 03E55, 03E45.

Key words and phrases: stationary reflection, extender model.

The author's research was funded in part by National Science Foundation, grant number DMS-0400467. 
Subcompactness is a large cardinal property. The author and others have asked whether there is a large cardinal axiom equivalent to Stationary Reflection at $\kappa^{+}$in $L[E]$. Because Stationary Reflection at $\kappa^{+}$fails in $L[E]$ if $\kappa$ is not subcompact, we may search for a characterization that applies only if $\kappa$ is subcompact. Ultimately, the solution depends on what we consider a large cardinal axiom. For example, is the following strong evidence of stationary reflection a large cardinal axiom?

DEFinition 1.2. $\kappa$ is an $S E S R$ cardinal iff for all stationary subsets $A$ of $\kappa^{+} \cap \operatorname{cof}(<\kappa)$, there exists $\mu<\kappa$ and an elementary embedding

$$
\pi:\left(H_{\mu^{+}}, a\right) \rightarrow\left(H_{\kappa^{+}}, A\right)
$$

with $\operatorname{crit}(\pi)=\mu$ such that $a \cap \sigma$ is stationary in $\sigma$ for some $\sigma<\mu^{+}$.

Obviously, if $\kappa$ is a subcompact cardinal, then $\kappa$ is an SESR cardinal $\Leftrightarrow$ Stationary Reflection holds at $\kappa^{+}$ independently of whether $V=L[E]$. Unfortunately, this solution feels like a cheat. Why? Perhaps because we are used to proofs of Stationary Reflection at $\kappa^{+}$from the existence of an elementary embedding with a discontinuity at $\kappa^{+}$. Solovay started with a cardinal $\kappa$ that is $\kappa^{+}$compact. More recently, Jensen used a quasicompact embedding, i.e., an embedding

$$
\pi:\left(H_{\kappa^{+}}, A\right) \rightarrow\left(H_{\lambda^{+}}, B\right)
$$

with $\operatorname{crit}(\pi)=\kappa$, to show that if $\tau=\sup \left(\pi\left[\kappa^{+}\right]\right)$and $A$ is stationary in $\operatorname{cof}(<\kappa) \cap \kappa^{+}$, then $B \cap \tau$ is stationary in $\tau$, so $A \cap \sigma$ is stationary in $\sigma$ for some $\sigma<\kappa^{+}$. Notice that $\operatorname{cf}(\sigma)=\mu^{+}$for some measurable $\mu<\kappa$ in this case. Arguments of this sort always seem to show something more than Stationary Reflection, at least as far as we can see.

We do not abandon our characterization of Stationary Reflection in terms of SESR cardinals. Rather, we try to make it more interesting. In Section 2, we look more closely at the characterization of $\square_{\kappa}$ in $L[E]$. It has a third component that we have not yet discussed. We will introduce an analogous third component to our characterization of Stationary Reflection. See Corollary 2.12.

In Section 3, we describe a concrete situation in which $\kappa$ is subcompact but there is a non-reflecting stationary subset of $\kappa^{+} \cap \operatorname{cof}(<\kappa)$. We show that this is the case in $L[E]$ if there is no cardinal $\mu<\kappa$ such that $\mu$ is a subcompact cardinal and a $\kappa$-strong cardinal. See Corollary 3.2. Cummings [2] obtained a related result using forcing. He showed that if $\kappa$ is a measurable subcompact cardinal, then there is a poset $\mathbb{P}$ such that in $V^{\mathbb{P}}, \kappa$ is a measurable subcompact cardinal and Dense Non-Reflection holds at $\kappa^{+}$. Relative consistency results like this are comforting because we have not yet constructed extender models with subcompact cardinals. 
Finally, in Section 4, we list some hypotheses that imply Stationary Reflection at $\kappa^{+}$and close with an open question.

2. Characterization in extender models. We begin with a string of definitions and results that outlines the characterization of $\square_{\kappa}$ in terms of subcompact cardinals.

Definition 2.1. Assume that $V=L[E]$. Let $S_{\kappa^{+}}$be the set of $\alpha<\kappa^{+}$ such that

- $E_{\alpha}$ is a superstrong extender,

- if $\mu=\operatorname{crit}\left(E_{\alpha}\right)$ and

$$
i: L[E] \rightarrow \operatorname{ult}\left(L[E], E_{\alpha}\right)
$$

is the ultrapower map, then $i(\mu)=\kappa$.

Observe that $S_{\kappa^{+}} \subseteq \kappa^{+} \cap \operatorname{cof}(<\kappa)$ if $V=L[E]$. This is because $\operatorname{cf}(\alpha)=$ $\mu^{+}<\kappa$ under the conditions of Definition 2.1.

Theorem $2.2([4])$. Assume that $V=L[E]$. Suppose that $S_{\kappa^{+}}$is not stationary in $\kappa^{+}$. Then $\square_{\kappa}$ holds.

No details of the proof of Theorem 2.2 will be used in this section.

Definition 2.3. Assume that $V=L[E]$. For each $A \subseteq \kappa^{+}$, let $T_{A}$ be the set of $\alpha \in S_{\kappa^{+}}$such that

$$
\left(J_{\alpha}^{E}, A \cap \alpha\right) \prec\left(J_{\kappa^{+}}^{E}, A\right)
$$

and, if $i: L[E] \rightarrow \operatorname{ult}\left(L[E], E_{\alpha}\right)$ is the ultrapower map, then

$$
A \cap \alpha \in \operatorname{ran}(i) .
$$

Proposition 2.4 (Jensen). Assume that $V=L[E]$. Suppose that $T_{A}$ $\neq \emptyset$ for all $A \subseteq \kappa^{+}$. Then $\kappa$ is a subcompact cardinal.

Proof. Let $\alpha \in T_{A}$ and $i: L[E] \rightarrow \operatorname{ult}\left(L[E], E_{\alpha}\right)=L[F]$ be the ultrapower map. Let $\mu=\operatorname{crit}(i)$ and $a=i^{-1}(A \cap \alpha)$. Then

$$
\left(H_{\mu^{+}}, a\right)=\left(J_{\mu^{+}}^{E}, a\right) \rightarrow\left(J_{\alpha}^{F}, A \cap \alpha\right)=\left(J_{\alpha}^{E}, A \cap \alpha\right) \prec\left(J_{\kappa^{+}}^{E}, A\right)=\left(H_{\kappa^{+}}, A\right)
$$

is an elementary embedding that witnesses subcompactness for $A$.

Proposition 2.5 (Jensen). Assume that $V=L[E]$. Suppose that $S_{\kappa^{+}}$ is stationary in $\kappa^{+}$. Then $T_{A} \neq \emptyset$ for all $A \subseteq \kappa^{+}$.

Proof. Suppose otherwise. Let $B$ be the $<_{L[E]}$-least $A \subseteq \kappa^{+}$such that $T_{A}=\emptyset$. Then $B$ is definable in $J_{\kappa^{++}}^{E}$. Since $S_{\kappa^{+}}$is stationary, there exists $\beta \in S_{\kappa^{+}}$such that

$$
\beta=\kappa^{+} \cap \operatorname{Hull}_{\kappa}^{J^{++}}(\beta) .
$$


Let

$$
\pi: J_{\gamma}^{F} \simeq \operatorname{Hull}_{\kappa}^{J^{++}}{ }^{E}(\beta)
$$

be the inverse of the Mostowski collapse. Then

$$
\pi(\beta)=\kappa^{+} \text {and } \pi(B \cap \beta)=B .
$$

Let

$$
j: L[E] \rightarrow \operatorname{ult}\left(L[E], E_{\beta}\right)
$$

be the ultrapower map. By the condensation lemma, $J_{\gamma}^{F}$ is an initial segment of $\operatorname{ult}\left(L[E], E_{\beta}\right)$. This justifies writing $L[F]$ for $\operatorname{ult}\left(L[E], E_{\beta}\right)$. Let $\nu=\operatorname{crit}\left(E_{\beta}\right)$. Note that

$$
j\left(\nu^{+}\right)=\beta=\left(\kappa^{+}\right)^{L[F]} .
$$

Since $\pi$ is elementary, $J_{\gamma}^{F}$ satisfies the sentence

$$
B \cap \beta \text { is the }<_{L[F]} \text {-least } A \subseteq \beta \text { such that } T_{A}=\emptyset .
$$

But this sentence is upward absolute from $J_{\gamma}^{F}$ to $L[F]$. So $B \cap \beta \in \operatorname{ran}(j)$, which is a contradiction.

Proposition 2.6 ([1]). If $\kappa$ is a subcompact cardinal, then $\square_{\kappa}$ fails.

The following characterization is an immediate consequence of Theorem 2.2 and Propositions 2.4-2.6.

Corollary 2.7. Assume that $V=L[E]$. Then the following are equivalent:

- $\kappa$ is subcompact.

- $\square_{\kappa}$ fails.

- $S_{\kappa^{+}}$is stationary in $\kappa^{+}$.

Now we work towards a characterization of Stationary Reflection at $\kappa^{+}$ along lines similar to Corollary 2.7.

Definition 2.8. Assume that $V=L[E]$. For each $A \subseteq \kappa^{+}$, let $S_{A}$ be the set of $\alpha<\kappa^{+}$such that

- $E_{\alpha}$ is a superstrong extender,

- if $\mu=\operatorname{crit}\left(E_{\alpha}\right)$ and

$$
i: L[E] \rightarrow \operatorname{ult}\left(L[E], E_{\alpha}\right)
$$

is the ultrapower map, then

$-i(\mu)=\kappa$,

- there exists $\sigma<\mu^{+}$such that

$$
\{\delta<\sigma \mid i(\delta) \in A\}
$$

is stationary in $\sigma$. 
Observe that $S_{\kappa^{+}}$really is $S_{A}$ in the case $A=\kappa^{+}$. And $S_{A} \subseteq S_{\kappa^{+}}$in general.

Proposition 2.9. Assume that $V=L[E]$. Let $A \subseteq \kappa^{+}$. Suppose that $S_{A}$ is stationary in $\kappa^{+}$. Then there exists $\tau<\kappa^{+}$such that $A \cap \tau$ is stationary in $\tau$.

Proof. Run the proof of Proposition 2.5 using $S_{A}$ instead of $S_{\kappa^{+}}$. This gives $\alpha \in S_{A} \cap T_{A}$. Then

$$
\left(J_{\alpha}^{E}, A \cap \alpha\right) \prec\left(J_{\kappa^{+}}^{E}, A\right)
$$

and, if $i: L[E] \rightarrow \operatorname{ult}\left(L[E], E_{\alpha}\right)$ is the ultrapower map, then

$$
A \cap \alpha \in \operatorname{ran}(i) \text {. }
$$

Let $\mu=\operatorname{crit}(i)$ and

$$
a=\left\{\delta<\mu^{+} \mid i(\delta) \in A\right\} .
$$

Then $i(a)=A \cap \alpha$ and there exists $\sigma<\mu^{+}$such that $a \cap \sigma$ is stationary in $\sigma$. Therefore, $i(a \cap \sigma)=A \cap i(\sigma)$ is stationary in $i(\sigma)$ in $J_{\alpha}^{E}$, hence in $L[E]$.

Proposition 2.10. Assume that $V=L[E]$. Suppose that $S_{\kappa^{+}}$is stationary in $\kappa^{+}$. Let $A \subseteq \kappa^{+}$. Suppose that $\tau<\kappa^{+}$and $A \cap \tau$ is stationary in $\tau$. Then

$$
T_{A}-\tau \subseteq S_{A}
$$

and $T_{A}$ is stationary in $\kappa^{+}$.

Proof. We may assume that $\tau$ is the least $\sigma<\kappa^{+}$such that $A \cap \sigma$ is stationary in $\sigma$. Towards seeing that $T_{A}-\tau \subseteq S_{A}$, consider an arbitrary $\alpha \in T_{A}-\tau$. Let $i: L[E] \rightarrow \operatorname{ult}\left(L[E], E_{\alpha}\right)=L[F]$ be the ultrapower map and $\mu=\operatorname{crit}(i)$. Because $\alpha \in T_{A}$,

$$
i(a)=A \cap \alpha \quad \text { where } \quad a=\left\{\delta<\mu^{+} \mid i(\delta) \in A\right\} .
$$

And $A \cap \tau$ is stationary in $\tau$ in $L[E]$, in $J_{\alpha}^{E}=J_{\alpha}^{F}$, hence in $L[F]$. By the minimality of $\tau$ and the elementarity of $i$, there exists $\sigma<\mu^{+}$such that $i(\sigma)=\tau$ and $a \cap \sigma$ is stationary in $\sigma$. Therefore $\alpha \in S_{A}$.

Now, to see that $T_{A}$ is stationary in $\kappa^{+}$, repeat the proof of Proposition 2.5 with a minor modification. Let $(B, D)$ be the $<_{L[E]}$-least pair $(A, C)$ of subsets of $\kappa^{+}$such that $C$ is club in $\kappa^{+}$and $T_{A} \cap C=\emptyset$. Find $\beta$ as in the proof of Proposition 2.5 with the additional property that $\beta \in D$. At the end of the proof, we find instead that $J_{\gamma}^{F}$ satisfies the sentence:

$(B \cap \beta, D \cap \beta)$ is the $<_{L[F]}$ least pair $(A, C)$ of subsets of $\beta$ such that $C$ is club in $\beta$ and $T_{A} \cap C=\emptyset$.

This sentence is upward absolute to $L[F]$ and so $B \cap \beta \in \operatorname{ran}(j)$. This shows that $\beta \in T_{B} \cap D$, which is the desired contradiction. 
Putting together Corollary 2.7 with Propositions 2.9 and 2.10, we obtain the following two characterizations. Keep in mind that in $L[E]$, Stationary Reflection at $\kappa^{+}$fails if $\kappa$ is not a subcompact cardinal.

Corollary 2.11. Assume that $V=L[E]$. Suppose that $\kappa$ is a subcompact cardinal. Then the following two conditions are equivalent for each $A \subseteq \kappa^{+}:$

- There exists $\tau<\kappa^{+}$such that $A$ is stationary in $\tau$.

- $S_{A}$ is stationary in $\kappa^{+}$.

Corollary 2.12. Assume that $V=L[E]$. Then the following three principles are equivalent:

- $\kappa$ is an SESR cardinal.

- Stationary Reflection at $\kappa^{+}$.

- For all stationary subsets $A$ of $\kappa^{+} \cap \operatorname{cof}(<\kappa), S_{A}$ is stationary in $\kappa^{+}$.

3. A non-reflecting stationary set. In this section, we describe a situation in which $\kappa$ is subcompact but there is a non-reflecting stationary subset of $\kappa^{+} \cap \operatorname{cof}(<\kappa)$.

Proposition 3.1. Assume that $V=L[E]$. Suppose that there is no $\mu<\kappa$ such that $\mu$ is a subcompact cardinal and a $\kappa$-strong cardinal. Then $S_{\kappa^{+}} \cap \tau$ is not stationary in $\tau$ for all $\tau<\kappa^{+}$.

Proof. For contradiction, suppose that $S_{\kappa^{+}} \cap \tau$ is stationary in $\tau$ and $\tau<\kappa^{+}$. We will borrow some terminology and notation from [4]. Let $N_{\tau}$ be the collapsing level for $\tau$.

CASE 1: $N_{\tau}$ is pluripotent. Let

$$
\mu=\operatorname{crit}\left(E_{\mathrm{top}}^{N_{\tau}}\right) .
$$

The hypothesis of Case 1 is exactly that $N_{\tau}$ is active and

$$
\mu<\kappa=\omega \varrho_{N_{\tau}}^{1} .
$$

Then $\mu$ is a $\kappa$-strong cardinal in $L[E]$ as witnessed by $E_{\text {top }}^{N_{\tau}}$. By the hypothesis of Proposition 3.1, $\mu$ is not a subcompact cardinal. Therefore $S_{\mu^{+}}$is not stationary in $\mu^{+}$. Let $C$ be a club subset of $\mu^{+}$with $C \cap S_{\mu^{+}}=\emptyset$. We may assume that if $\vartheta \in C$, then $\mu$ is the largest cardinal of $J_{\vartheta}^{E}$ since the set of such $\vartheta$ is also club in $\mu^{+}$.

Consider an arbitrary $\vartheta \in C$. Let

$$
i: L[E] \rightarrow \operatorname{ult}\left(L[E], E_{\mathrm{top}}^{N_{\tau}}\right)
$$

be the ultrapower map. Let $\sigma_{\vartheta}$ be the supremum of ordinals $<\tau$ of the form $i(f)\left(a, d_{N_{\tau}}\right)$ for some $f \in N_{\vartheta}$ and $a \in[\kappa]^{<\omega}$. Here $d_{N_{\tau}}$ is the Dodd 
parameter of $N_{\tau}$. The function $\vartheta \mapsto \sigma_{\vartheta}$ is continuous from $C$ to $\tau$ because we need only consider functions

$$
f:[\mu]^{<\omega} \times[\mu]^{\left|d_{N_{\tau}}\right|} \rightarrow \mu
$$

in the definition of $\sigma_{\vartheta}$. That is, just functions in

$$
J_{\vartheta}^{E}=\bigcup_{\vartheta^{\prime} \in C \cap \vartheta} J_{\vartheta^{\prime}}^{E}
$$

if $\vartheta \in \lim (C)$. In addition,

$$
\tau=\sup _{\vartheta \in C} \sigma_{\vartheta}
$$

The calculations in [4] show that there exists $\vartheta_{0}<\mu^{+}$such that for all $\vartheta \in C-\vartheta_{0}$,

$$
N_{\sigma_{\vartheta}}=\operatorname{ult}\left(N_{\vartheta}, E_{\mathrm{top}}^{N_{\tau}}\left\lceil\left(\kappa \cup d_{N_{\tau}}\right)\right) .\right.
$$

We claim that if $\vartheta \in C-\vartheta_{0}$, then $\sigma_{\vartheta} \notin S_{\kappa^{+}}$. For let $\pi: N_{\vartheta} \rightarrow N_{\sigma_{\vartheta}}$ be the ultrapower map. For contradiction, suppose that $\sigma_{\vartheta} \in S_{\kappa^{+}}$. Then

$$
N_{\sigma_{\vartheta}}=J_{\sigma_{\vartheta}}^{E}
$$

and $E_{\sigma_{\vartheta}}$ is a superstrong extender that sends its critical point up to $\kappa$. It follows that $\pi(\mu)=\kappa, N_{\vartheta}=J_{\vartheta}^{E}$ and $E_{\vartheta}$ is a superstrong extender that sends its critical point up to $\mu$. Thus $\vartheta \in S_{\mu^{+}}$, which is a contradiction.

Therefore $\left\{\sigma_{\vartheta} \mid \vartheta \in C-\vartheta_{0}\right\}$ is a club subset of $\tau$ that is disjoint from $S_{\kappa^{+}}$.

CASE 2: Otherwise. By imitating Jensen's proof of $\square_{\kappa}$ in $L$, the authors of [4] give an argument that shows that there exists a club subset $C$ of $\tau$ such that $N_{\sigma}$ is not pluripotent for all $\sigma \in C$. In particular, $\sigma \notin S_{\kappa^{+}}$for all $\sigma \in C$.

Corollary 3.2. Assume that $V=L[E]$. Suppose that $\kappa$ is a subcompact cardinal but there is no $\mu<\kappa$ such that $\mu$ is a subcompact cardinal and a $\kappa$-strong cardinal. Then $S_{\kappa^{+}}$is a non-reflecting stationary subset of $\operatorname{cof}(<\kappa) \cap \kappa^{+}$.

4. Upper bounds. In this section, we list some hypotheses on $\kappa$ that imply that $\kappa$ is an SESR cardinal.

Definition 4.1 (Jensen). $\kappa$ is a quasicompact cardinal if for each $A \subseteq \kappa^{+}$, there exist $\lambda>\kappa, B \subseteq \lambda^{+}$and an elementary embedding

$$
\pi:\left(H_{\kappa^{+}}, A\right) \rightarrow\left(H_{\lambda^{+}}, B\right)
$$

with $\operatorname{crit}(\pi)=\kappa$.

Proposition 4.2 (Jensen). Suppose that $\kappa$ is quasicompact. Let $A$ be a stationary subset of $\kappa^{+} \cap \operatorname{cof}(<\kappa)$. Then there exists $\sigma<\kappa^{+}$such that $A \cap \sigma$ is stationary in $\sigma$. 
The proof was sketched in the introduction. In particular, quasicompact cardinals are SESR cardinals. Here are some refinements of Proposition 4.2.

Proposition 4.3. Let $A$ be a stationary subset of $\kappa^{+} \cap \operatorname{cof}(<\kappa)$. Suppose that $E$ is an extender and

$$
j: V \rightarrow \operatorname{ult}(V, E)=M
$$

is the ultrapower map. Assume

$$
H_{\kappa^{+}} \cup\{A\} \subseteq M
$$

and there is an elementary embedding

$$
\pi:\left(H_{\kappa^{+}}, A\right) \rightarrow\left(\left(H_{\lambda^{+}}\right)^{M}, B\right)
$$

with $\operatorname{crit}(\pi)=\kappa$ such that $B, \pi \in M$. Then there exists $\sigma<\kappa^{+}$such that $A \cap \sigma$ is stationary in $\sigma$.

Notice that if the hypotheses of Proposition 4.3 are satisfied, then they are satisfied by an extender $E$ with length $(E)<\left(2^{\kappa}\right)^{+}$. Also, there is nothing in the hypotheses that rules out that $E$ is a normal measure over $\kappa$.

Proposition 4.4. Let $\kappa$ be a subcompact cardinal and $A$ be a stationary subset of $\kappa^{+} \cap \operatorname{cof}(<\kappa)$. Suppose that $U$ is a normal measure over $\kappa$ and there is a function

$$
f: \kappa \rightarrow H_{\kappa^{+}} \text {such that } A=[f]_{U} .
$$

Assume that there exists $X \in U$ such that for all $\alpha \in X$, there exist $\beta$, $b \subseteq \beta^{+}$and an elementary embedding

$$
\pi:\left(H_{\alpha^{+}}, f(\alpha)\right) \rightarrow\left(H_{\beta^{+}}, b\right)
$$

with $\operatorname{crit}(\pi)=\alpha$. Then there exists $\sigma<\kappa^{+}$such that $A \cap \sigma$ is stationary in $\sigma$.

As we mentioned in the introduction, Cummings [2] has shown that if $\kappa$ is a measurable subcompact cardinal, then there is a forcing notion that preserves this large cardinal hypothesis and forces Dense Non-Reflection at $\kappa^{+}$. Several open questions remain. For example, is it consistent for $\kappa$ to be a measurable subcompact cardinal such that, for all $A \subseteq \kappa^{+}$, there is a normal measure $U$ with $A \in \operatorname{ult}(V, U)$, but Dense Non-Reflection holds at $\kappa^{+}$?

\section{References}

[1] D. Burke, Generic embeddings and the failure of box, Proc. Amer. Math. Soc. 123 (1995), 2867-2871.

[2] J. Cummings, Compactness and incompactness phenomena in set theory, in: 2001 Logic Colloquium (Vienna), to appear. 
[3] E. Schimmerling and M. Zeman, Square in core models, Bull. Symbolic Logic 7 (2001), 305-314.

[4] - - - Characterization of $\square_{\kappa}$ in core models, J. Math. Logic 4 (2004), 1-72.

Department of Mathematical Sciences

Carnegie Mellon University

Pittsburgh, PA 15213, U.S.A.

E-mail: eschimme@andrew.cmu.edu

Received 10 April 2005;

in revised form 2 August 2005 